CAHIERS DE

NARRATOLOGIE

\section{Cahiers de Narratologie}

Analyse et théorie narratives

29 | 2015

Street Art 1

\title{
Arturo Pérez-Reverte, El francotirador paciente. Lectures du graffiti et de l'art urbain
}

\section{Christine Di Benedetto}

\section{(2) OpenEdition}

\section{Journals}

\section{Electronic version}

URL: http://journals.openedition.org/narratologie/7422

DOI: $10.4000 /$ narratologie. 7422

ISSN: 1765-307X

Publisher

LIRCES

\section{Electronic reference}

Christine Di Benedetto, « Arturo Pérez-Reverte, El francotirador paciente. Lectures du graffiti et de l'art urbain », Cahiers de Narratologie [Online], 29 | 2015, Online since 27 January 2016, connection on 19 April 2019. URL : http://journals.openedition.org/narratologie/7422 ; DOI : 10.4000/narratologie.7422

This text was automatically generated on 19 April 2019

Article L.111-1 du Code de la propriété intellectuelle. 


\title{
Arturo Pérez-Reverte, El francotirador paciente. Lectures du graffiti et de l'art urbain
}

\author{
Christine Di Benedetto
}

1 Dans quelle mesure, à l'heure de s'interroger sur ce qu'est l'art urbain dans ses éventuelles constantes définitoires, ses évolutions temporelles et ses spécificités spatiales, la littérature pouvait-elle apporter des éléments? C'est la question que nous avons voulu poser en nous intéressant à une œuvre du romancier espagnol Arturo PérezReverte, El francotirador paciente ${ }^{1}$ (2013) - en France, La patience du franc-tireur-, qui, outre qu'il en affirme l'intérêt, offre des lectures de l'expression urbaine en partant de l'un de ses aspects, le graffiti.

2 Membre de la Real Academia Española, cet auteur associe depuis ses débuts récit d'aventures et peinture sociale. Il est d'abord connu pour des romans historiques dont l'action se déroule majoritairement au siècle d'Or espagnol. Puis il s'approche progressivement des questions directement contemporaines et internationales. Par ailleurs, fouillant à plusieurs reprises avec mots et univers fictionnels des problématiques d'ordre iconographique, il aborde l'univers de la peinture dès La tabla de Flandes (1990). Après avoir, dans El pintor de batallas (2006), interrogé la question de la reproduction des conflits armés, qu'elle soit photographique ou picturale, le romancier-chroniqueurreporter de guerre envisage dans son dernier roman un autre type de productions visuelles en forte prise avec son présent, le graffiti. Dans $\mathrm{El} \mathrm{francotirador} \mathrm{paciente,} \mathrm{il} \mathrm{associe}$ ainsi récit d'investigation, peinture d'une population urbaine marginale et l'une des manifestations les plus ambivalentes de l'art contemporain, l'art urbain. Il est clair que ce même terme recouvre un ensemble de réalités ; celles-ci se veulent souvent antisystème et illégales, mais leurs expressions peuvent être récupérées, autorisées, voire souhaitées par les instances administratives. Ici, c'est le monde du tag ou du graffiti, de ses émanations et ses limites, qui interpelle Arturo Pérez-Reverte, jusqu'à l'amener à questionner le concept d'art contemporain. 
3 En le choisissant comme décor pour un thriller dans lequel les morts mystérieusement téléguidées se succèdent et où il s'agit classiquement d'en démasquer l'instigateur et de le placer face à ses responsabilités, l'auteur fait découvrir au grand public un univers méconnu. Mais également, en lui donnant l'occasion d'y pénétrer, soutenu par des éléments narratifs organisés à cet effet, il le guide vers une vision qui, sans être manichéenne, accepte de considérer ce monde étranger et nié comme existant dans un espace urbain partagé.

4 Nous verrons donc comment s'organisent textuellement la diffusion des informations de base et la mise en place d'une capacité de différenciation et de nuance, à partir de pistes réflexives et d'une accumulation d'exemples, pour parvenir à l'acceptation du questionnement ${ }^{2}$.

5 Le modèle fictionnel permet à la fois d'aborder indirectement, donc plus facilement, un monde interdit, marqué par une double dialectique de la répulsion, mais aussi de façon inattendue d'y pénétrer individuellement. Comme le montre Vincent Jouve dans L'effetpersonnage dans le roman ${ }^{3}$, la réception des personnages étant programmée par le texte, le lecteur est ouvert à plusieurs niveaux de réception, désignés respectivement par les termes de lisant, lu et lectant. La réception ouvre d'abord sur une illusion mimétique que le lecteur accepte et par laquelle il parvient à ressentir les sensations des acteurs. En même temps, est mobilisée sa position de voyeur qui l'autorise à observer le danger sans risque pour lui-même. Enfin le jeu de déchiffrage de l'énigme entraîne en parallèle l'effort de compréhension de ce qui se joue dans un univers souvent rejeté en bloc.

\section{Décor signifiant pour une investigation multiple}

6 Alejandra Varela, surnommée Lex, est une journaliste amoureuse de littérature, sorte de chercheuse cultivée au flair précieux ${ }^{4}$. Elle est aussi spécialiste de l'art urbain avec à son actif une thèse universitaire sur le graffiti. Elle se trouve lancée par un éditeur, Mauricio Bosque, sur la piste de «Sniper », un artiste reconnu dans le monde du graffiti, sorte de légende vivante mais qui entretient farouchement son anonymat. Caché depuis deux années, sa tête mise à prix, il est l'artiste le plus célèbre et le plus recherché de l'art urbain, « à mi-chemin entre Banksy et Salman Rushdie... », annonce le texte (LPF, p. 215). Il a commencé sa carrière en tant que simple graffeur, vingt ans auparavant, et n'a presque jamais été vu depuis qu'il est devenu une sorte de «marque déposée et point final : Sniper. Le franc-tireur solitaire. » (LPF, p. 236).

7 Pour le retrouver, elle accepte de devenir enquêtrice, une « scout » en langage du métier ( LPF, p. 18). La maison d'édition entend en effet publier un ouvrage d'art exceptionnel. Suivi d'expositions et d'une commercialisation au niveau mondial, il ouvrirait la voie à une reconnaissance institutionnelle, et bien entendu économique, du graffeur. Choisi comme faisant partie des meilleurs et donc admis à figurer dans le monde de l'Art, celuici jouirait d'une garantie de célébrité et postérité : «moi je le rends immortel en faisant entrer son œuvre dans le cercle des dieux » (LPF, p. 227).

Deux univers coexistent, visibles dès ce projet initial, celui de la nuit, de la marge, de l'antisystème, et une société pour laquelle tout est économie, jusqu'à la rébellion. La passerelle entre les deux est le monde de l'art qui, un jour, décide de s'intéresser à ce qui n'est au départ pas fait pour une quelconque marchandisation, le graffiti et ses émanations. Arturo Pérez-Reverte ne se contente déjà pas, on le voit, de les placer comme 
simple décor de son enquête. Il interroge dès l'incipit du roman non seulement le quoi mais aussi le pourquoi : que se passe-t-il pour qu'un graffeur isolé et mystérieux fasse école et soit ensuite recherché et valorisé par les collectionneurs et les musées?

L'enquête elle-même se complique et devient duelle car le même Sniper s'avère être l'initiateur d'actions urbaines spectaculaires auxquelles participent d'autres auteurs de graffitis. S'ils le font de manière volontaire, il n'en reste pas moins que l'inflation dans les sollicitations et le danger croissant de celles-ci conduit au décès de plusieurs jeunes. Et le richissime père de l'un d'entre eux, Lorenzo Biscarrués, engage également deux détectives pour suivre Lex afin de s'appuyer sur elle pour retrouver le leader et obtenir vengeance, puisque Daniel, son fils de 17 ans, s'est tué devant sa porte en effectuant une chute de 78 mètres pendant qu'il tentait d'apposer sa signature, « Holden », sur un toit incliné à $45^{\circ}$.

Une double poursuite s'établit donc, au sein d'un labyrinthe, nocturne souvent, dans lequel interviennent des acteurs qui forment un réseau aux interactions hasardeuses et qui laisse progressivement filtrer des informations tout en déroulant amplement un matériau documentaire et analytique. Lex, à la fois chasseur et proie, mais aussi vecteur de connaissance et de réflexion va, selon l'expression de Norman Mailer, occuper une place d'« enquêteur esthétique ${ }^{8}$ » dans le monde du graffiti.

\section{Contours : acteurs et fonctionnement}

11 Pour répondre à la question initiale de ce qu'est qu'un graffeur, il convient de débusquer à la fois qui il est et ce qu'il cherche, éléments que le texte n'aura de cesse de préciser tout au long de ses 300 pages.

12 Dès l'épigraphe figurent quatre lignes d'un graffeur new-yorkais, Ken, sur un mur, en 1986. Supposées être émises dans une réalité extratextuelle, elles posent d'emblée une première interrogation, celle du où ? Qu'en est-il de la trace de ce qui est éphémère, de la conservation pour ce qui n'est le plus souvent pas répertorié et figure rarement dans les catalogues des bibliothèques? Elles donnent également le ton et établissement les termes du débat:

Il était une fois une race particulière d'individus

Qu'on appelait les écrivains de graffitis.

Ils ont livré un combat féroce contre la société.

On n'en connaît pas encore le résultat ${ }^{9}$.

Reprenant l'incipit des contes populaires, dans un univers atemporel, la spécificité et la noblesse des héros, ici nommés « écrivains de graffiti » sont mises en évidence. Il semble s'agir d'une espèce rare, qui engagée dans un combat, se dresse contre un ennemi imprécis, appelé « la société ». Ainsi, au seuil de l'œuvre, s'initie la mythification que le roman construira en neuf chapitres, assortis de ce qui peut apparaître comme une introduction.

Celle-ci annonce dès son titre l'univers spatio-temporel de ce phénomène, urbain et contemporain: "Dans la ville. 1990 ». Menée par un narrateur hétérodiégétique, contrairement aux chapitres dont la voix narrative est celle de l'enquêtrice, elle offre une focalisation générale et omnisciente sur un univers complexe. Dans un cadre déjà fictionnel, le narrateur expose les éléments donnés comme non discutables d'un monde fondé sur le besoin d'être vu, lu et entendu, pour une affirmation d'existence. Guidé par 
l'obtention du respect à travers des rivalités dans des mondes marginaux, il intègre des codes expressifs balisant une démarche qui exige de se situer face au danger et fait apparaître héros et traîtres.

Une catégorie donc, un tout, dont l'ennemi commun est l'ordre établi, au-delà des frontières de la légalité où le graffiti se développe. Il s'agit de faire face à l'interdit, au danger représenté par gardiens ou policiers: ne jamais rester longtemps au même endroit, écrire rapidement et s'en aller. Mais cette catégorie qui semble homogène recouvre des différences et des castes car il s'agit d'abord de trouver sa place dans ce qui, de l'extérieur seulement, apparaît comme une communauté uniforme.

Dans un double mouvement de déclaration d'intention pour soi-même et de reconnaissance du statut conférée par l'Autre, l'introduction propose une avancée dans la définition, par une série de caractéristiques :

Il s'agit d'abord d'un univers nocturne, urbain et clandestin ${ }^{10}$. Jeune aussi, car ses vétérans ont tout juste vingt ans, et marqué par une consommation de boissons standardisées (Coca-Cola) et de musiques actuelles (break dance, rap). Caractérisent ses membres le port de vêtements neutres destinés à camoufler, comme pour la guerre ou la chasse: jeans, sweat-shirts à capuche noirs, chaussures de sport. Une sorte d'uniforme que complètent des accessoires indispensables: un sac à dos taché de peinture rempli d'aérosols avec embouts différents selon le type de besoin.La connivence visuelle évoque un fonctionnement collectif, qu'il soit un agrégat d'individualités ou, ponctuellement, de bandes. Sans se connaître au préalable, ils se reconnaissent par leur ipséité : «à leurs sacs et leur allure » et un signe : «le geste de peindre quelque chose» (LPF, p. $\left.{ }^{11}\right)$.

Il est primordial de se situer face aux membres de la même espèce par son mérite dans une hiérarchie invisible qui structure ce monde apparemment hors-la-loi dont :

on ne gravissait les échelons que par les mérites acquis en solitaire ou en petits groupes, chacun imposant son nom de guerre à force de persévérance, le multipliant à l'infini aux quatre coins de la ville (LPF, pp. 9-10 12 ).

Les deux personnages qui apparaissent dans l'introduction ne sont que : «des plébéiens, de simples fantassins. L'échelon le plus bas de leur tribu urbaine. Parias d'une société individualiste » qui cherchent à se situer et à exister en se faisant une réputation, face à des « œuvres d'auteurs consacrés, de rois de la rue » (LPF, pp. 9-1013).

La synthèse extrême comme mode d'expression; entre vandalisme, cri de protestation et œuvre d'art

L'action est toujours celle d'écrire :

sur un mur, un véhicule, le rideau de fer d'un magasin (...) cherchant ensemble des espaces vides ou des pièces laissées par d'autres bombeurs sur des murs saturés, usines abandonnées de la proche banlieue et gares de triage (LPF, p. $\left.{ }^{14}\right)$.

21 Et l'écriture est indissociable d'un espace, « une écriture en conditions » (LPF, p. 12).

22 Deux zones-cibles sont prioritaires: les lieux fixes ou mouvants de la ville (murs, magasins, transports), les non-lieux urbains, ou lieux abandonnés de la périphérie et de l'ère post-industrielle, tels que des usines hors-service.

Le texte effectue une présentation d'abord didactique du tag (surnom, lettres) à partir d'exemples. Il définit des nuances, des simples signatures au marqueur, « hâtives et sans grand talent artistique, misant plus sur la quantité que sur la qualité » (LPF, p. $\left.23^{15}\right)$ à l'expression plus rare quand l'auteur a plus de temps ou de tempérament, et que la calligraphie du graffiti occupe plus d'espace et demande des aérosols pour la couleur. 

code couleur à Madrid par exemple ${ }^{17}$, ou l'accord implicite pour respecter les monuments publics, ne pas écrire sur le graffiti d'un autre... Mais de ces normes, comme de toutes, peuvent surgir d'autres transgressions. niveau qui est le fait en soi d'écrire, le second est la surcharge. La surface s'apparente à un palimpseste; il faut effacer pour y réécrire. Dans un rapport de force où le lieu n'est pas le support mais l'enjeu, il s'agit soit de trouver un espace libre, un trou, pour y apposer sa marque, soit d'oser la surimpression : «toyer le tag d'un des grands » (LPF, p. $\left.10^{18}\right)$. Mais l'acte n'est pas anodin : « bomber sur la signature d'un autre était toujours une déclaration de guerre; la violation d'un nom, d'un territoire, d'une renommée qui n'étaient pas les leurs 》 (LPF, p. $\left.10^{19}\right)$ et met en évidence une violence interne ${ }^{20}$. L'affichage de soi se fait par la signature. Dans l'introduction, le nom de l'équipe est un sigle, DQTN, en soi une déclaration de combat: "Devine Qui T'a Niqué» (LPF, p. 12 ${ }^{21}$ ). L'argot et la déformation orthographique - que la traduction française perd par rapport à l'espagnol (dans AKTJ : kién pour quién) -, renvoient à la génération SMS, mais cela demeure caché derrière des lettres seulement comprises par les initiés. Le nom, c'est la « foi du graffiti ", dit Norman Mailer, les «vaguelettes de l'ego ${ }^{22}$ ». Dans le surnom ou le nom de guerre choisi, l'exhibition de soi se fait avec un anonymat partiel: il s'agit d'être incarné, reconnu sans être connu.

$\mathrm{Si}$, au regard de l'ordre urbain établi, cette saturation illégale de l'espace public avec des signatures peut être considérée comme du vandalisme : « sans souci de style, sans respect pour rien ni personne » (LPF, p. 10 $\left.10^{23}\right)$, en réalité les enjeux que recouvre la production du graffiti se situent entre cri de protestation et affirmation d'existence ${ }^{24}$. La signature se décline comme un synonyme de vie, un cri de désespoir de quelqu'un qui n'est personne : «le nom de Personne multiplié jusqu'à l'infini » (LPF, p. $30^{25}$ ), sera-t-il dit plus tard à propos de la graffeuse Lita, alors qu'il est encore plus difficile d'être une fille dans cet univers masculin. Dans une fusion des sens, l'odorat et l'ouïe sont stimulés; l'intérieur de l'individu se retrouve en accord avec l'extérieur ; l'un devient un tout : «Odeur de gloire urbaine, de liberté illégale, de renommée dans l'anonymat. Avec de grands déferlements, boum, boum, boum, d'adrénaline " (LPF, p. $12^{26}$ ). Quelques adjectifs sonnent comme un manifeste de ce mouvement sans chef : urbain, libre, illégal, célèbre, anonyme, fugace, excitant...

Dans l'introduction donc, en deux pages, l'univers du graffiti est brossé, plutôt positivement connoté, évoquant les principaux enjeux qui seront développés par la suite, d'un entre-soi conflictuel à un tous-contre-les-autres unitaire.

$\mathrm{Au}$ niveau de la diégèse, la présence de deux tagueurs anonymes, mais emblématiques avec leurs rêves et combats, permet la première rencontre avec le personnage principal, dont le nom clôt l'introduction. Cela lui donne ainsi formellement un relief proportionnel à sa place dans la hiérarchie interne des graffeurs et dans le système des personnages du roman : Sniper. Auparavant, sur presque trois pages, le narrateur décrit minutieusement non pas l'individu, toujours marqué par une profonde indéfinition (quelqu'un, de dos...) et qui semble passer au deuxième plan par rapport à sa production, mais la signature de celui-ci. Pour lui, le geste est aussi important que le résultat. De manière très visuelle, suivant les mouvements et détaillant les couleurs, le lecteur participe en focalisation interne à la réalisation matérielle du tag: six lettres d'un nom en couleurs, et le point du $\mathrm{i}$ en forme de cible: un rond traversé par une croix en forme de croix celte, devenant le 
viseur d'une arme, d'un fusil à télescope, utilisé pendant les guerres et guérillas urbaines par les francs-tireurs, les tireurs embusqués. De l'avis des observateurs avisés: «beaucoup plus que le simple travail d'un banal graffeur. C'était une œuvre exécutée dans toutes les règles de l'art» ( $\left.L P F, p .13^{27}\right)$, le maximum recherché dans la rue. La signature se pose toujours comme élément central. Mais quand le talent calligraphique s'ajoute à l'idée, alors l'œuvre affleure et on passe de la simple signature à la pièce. L'univers commence à générer ses propres mythes.

Une fois le contexte posé, au-delà de la production, c'est la fonctionnalité qui va être interrogée : le graffeur peut-il être considéré comme un écrivain, utilisant les surfaces urbaines comme des feuilles, la synthèse extrême comme mode d'expression? C'est ce que le roman va tenter de creuser autour du personnage de Sniper, recherché puis rencontré, et des nombreux acteurs qui en indiquent la piste et signifient, chacun, un état de cette population et de la variété de ses réalisations.

Une réflexion d'ordre esthétique intervient: à quel moment passe-t-on de graffeur, écrivain ou auteur de graffiti, à celle d'artiste de rue? Si le street art ne peut jouir d'une définition définitive car il est en permanente évolution, il est une catégorie plus ouverte et intégrante que le graffiti. Le premier est actuellement un enjeu de la gentrification des centres des villes, alors que le second reste dans le rejet, n'accepte pas de limite spatiale, inclut toujours l'idée de danger et est éphémère, temporaire, destiné à être effacé ou recouvert, au mieux à s'user. Une question commune demeure toutefois: celle de la surface et de sa propriété. A priori caractérisés par l'illégalité, l'absence de financement, l'autonomie par rapport au marché de l'art, la prise de décision individuelle quant à l'espace choisi et au motif apposé, le street art accepte des évolutions et des aménagements quand le graffiti s'affirme plus radicalement. Il peut toutefois s'étoffer, être reconsidéré a posteriori, ce qu'exprime le texte avec les productions du personnage de Sniper. Est susceptible d'en découler aussi une possibilité de récupération commerciale, avec ouverture de boutiques, customisation d'objets etc. On le voit, les frontières sont poreuses.

31 Le roman nourrit l'indétermination, construit la connotation positive du graffiti, en mettant en évidence son autonomie à travers le choix des formes, matières, styles et surtout des lieux et codes. Il souligne la capacité d'imposer ses propres normes en dépit de la réduction initiale du sujet à une signature, soulignant l'attention portée au matériau, le goût pour l'expérimentation formelle, la complémentarité entre forme et substance. Comme le souligne Richard Shusterman à propos de la culture populaire, le graffiti dans la ville fabrique une tradition spécifique basée sur la non-séparation de l'expression artistique et de la vie réelle. Il est manifestation d'existence de certains de ses habitants, habillage des lieux urbains et conquête des espaces; par conséquent la vie est la matière de cette expression artistique. De plus il "peut être esthétiquement légitimé par les expériences qu'il procure et les pratiques d'écoute, de vision et de critique qu'il engendre ${ }^{28} \%$. Ainsi unité et complexité, polysémie, ouverture et expérimentation formelle caractérisent-elles au fil du texte le monde du graffiti. 


\section{Spatialisation du récit : une écriture par touches répétées et tâtonnements dans un labyrinthe urbain}

L'axiologie du texte est construite grâce à la fonction de régie qu'exerce Lex, la narratrice autodiégétique, au long des neuf chapitres ${ }^{29}$ avec une double mise en espace.

En matière d'espace textuel, tous les outils de la temporalité sont mobilisés - moment de la narration, ordre du récit, rythme et fréquence - pour soutenir un même objectif, faire une place essentielle à la compréhension des phénomènes liés au graffiti. Le récit est ultérieur pendant toute l'enquête, mais devient simultané lors du dénouement, accentuant la pression vers la catharsis finale. Il suit linéairement les investigations de la protagoniste, dans une succession d'étapes logiques et concordantes, comme un spectateur en quête d'indices évoluerait dans la ville à la recherche de pistes et de productions cachées. Seules quelques analepses retracent les parcours de Sniper et de Lita, amante de la narratrice, dont le traitement narratif identique laisse deviner l'importance qu'elle aura au niveau de la diégèse. En ce qui concerne le rythme, alternent effets d'accélération à chaque progrès de l'enquête et au contraire de ralenti, lorsque, quasiment à chaque page, sont intégrées des pauses descriptives et analytiques. Enfin, le récit est souvent "singulatif anaphorique", selon l'expression de Gérard Genette, détaillant dès que possible les productions et de leurs enjeux; il est aussi «itératif ", revenant à de très nombreuses reprises sur les possibles contours de l'expression urbaine et la question de l'art contemporain. Enfin le pluri-perspectivisme peut figurer symboliquement la duplication des motifs propre au graffiti.

En complément de cette organisation intellectuelle, le lecteur a accès au ressenti physique de la narratrice en position de graffeuse, à deux occasions lorsqu'au cours de ses recherches elle se trouve elle-même à l'œuvre. On découvre de l'intérieur cette sensation en focalisation interne réduite aux émotions :

C'était donc ça (...). L'excitation intellectuelle, la tension physique, le défi lancé à sa propre sécurité, le contrôle des sensations et des émotions, l'immense euphorie de se mouvoir en pleine nuit, en plein danger, en transgressant l'ordre établi ou prétendu tel. En se déplaçant avec des précautions de soldat aux limites étroites du désastre. Sur le fil incertain du rasoir. (LPF, p. $205^{30}$ ).

Dominent les informations sensorielles, olfactives et auditives qui incitent à avoir du respect pour l'action narrée.

Dans une volonté de former le regard, le roman s'intéresse ensuite à la validité du graffiti en soi, entre les écueils d'une critique systématique ou d'une louange injustifiée.

Après les premières descriptions faites par les soldats $d u$ bas de l'échelle dans l'introduction, le récit intègre trois extraits plus académiques de la thèse de Lex qui s'interroge également sur la nature du graffiti et son évolution, ses liens avec la musique, mentionne des références et sources éditoriales, comme si l'auteur implicite fournissait au lecteur de quoi se renseigner au-delà du roman et prouvait la véracité de son contexte et de ses problématiques. S'ébauche une tentative de délimitation des contours du graffiti au sein de l'art urbain, sans en ignorer les détours et les points communs avec d'autres activités plastiques de rue : « l'agressivité de son caractère individualiste, voué à la rue, à la transgression et à la clandestinité. » (LPF, p. 45 $\left.{ }^{31}\right)$. 
d'objets reliés entre eux avec l'intention de provoquer. Les «infiltrations », par exemple, consistent à placer clandestinement des œuvres personnelles dans des musées ou expositions publiques. La référence extratextuelle est posée ; cela se produit au même moment où Banksy, «le célèbre graffeur de Bristol, commençait à faire un peu la même chose en Angleterre.» (LPF, p. 3855). Elles demeurent quelques heures, une journée au maximum quand le texte prend le temps de développer plusieurs exemples induisant un décalage entre la fugacité du fait rapporté et le soin mis à le décrire. Sniper reçoit alors le qualificatif de "terroriste de l'art», inventé par un critique et qui se diffuse dans les médias. En réaction, les invitations se multiplient. À Madrid où la mairie l'invite à intervenir dans une exposition de peinture officielle à l'air libre dans une enceinte industrielle de la périphérie réservée à cet effet : « art urbain, nouvelles tendances et tutti quanti » (LPF, p. 3936), ou à Barcelone, près du MACBA avec garantie de conservation de la pièce.

Ce qui est vu comme synonyme de soumission au système est refusé par Sniper. L'enjeu est une nouvelle fois rappelé : le graffiti est bien une question de vie et de mort, physique mais surtout mentale.

La lancinante question de l'art est abordée sous plusieurs facettes, rejeté dès la simple mention du terme : «Le mot artiste avait été prononcé avec un dédain corporatiste » (LPF, p. $\left.170^{37}\right)$. Le jugement du protagoniste est sévère :

Même l'art des rues est devenu, du fait des municipalités, un parc thématique (...). Ce concept imbécile de participation du public, si politiquement correct, a fini par n'être qu'un divertissement de plus (...). Mais moi je démontre que ça n'a rien d'amusant. Et que parfois il y a va de la vie. (LPF, p. $\left.246^{38}\right)$.

Cahiers de Narratologie, 29 | 2015 
Dans un refus d'entrer dans le jeu de l'art de rue "domestiqué », pendant cinq nuits consécutives, il barbouille toutes ses pièces avec sa seule signature (autotachado) et les accompagne de l'expression de sa philosophie sur un bus touristique de la ville: «L'Évangile en quatorze putains de mots. Sur un côté du bus il a écrit: Si c'est légal, c'est pas un graffiti. Et sur l'autre : Les rats ne font pas de claquettes. » (LPF, p. 40 $\left.{ }^{39}\right)$, ce qui est le titre du chapitre, consacré donc à l'affirmation du quoi et du comment, puis explicité lorsque la même expression est reprise avec l'élucidation de la métaphore : «Le pouvoir tente toujours de domestiquer ce qu'il ne peut contrôler » $\left(L P F\right.$, p. $\left.75^{40}\right)$. Il préfère dès lors qu'on l'appelle vandale qu'artiste.

Dans le même temps, pour vivre il crée des objets à vendre mais sans les signer : "Je dessine des pochettes de $C D$, des tatouages, je personnalise des fringues, je décore avec les gobetti un magasin par-ci par-là... Je me débrouille. » (LPF, p. 244 $\left.{ }^{41}\right)$.

Mais, seul maître de ses créations, Sniper s'insurge lorsqu'un galeriste d'Amalfi, Zuppa, décide à son compte de recueillir et vendre 17 pièces de ses pièces, tranquillement protégé par la volonté d'anonymat de l'auteur qui l'empêche d'en revendiquer la paternité. L'unique réponse possible de l'écrivain de rues est une action musclée visant à la destruction par l'acide des pièces avec une inscription : «Eso es lo que no soy, "ça, c'est ce que je ne suis pas" » (LPF, p. 13942), titre de ce même chapitre.

50 La double enquête est le moyen mis en place au sein du récit pour effectuer cette circulation spatiale et temporelle, mais également pour produire de multiples rencontres. Chacune apporte sa part d'informations sur ce que fait Sniper, mais surtout sur ce qu'est ce monde dans toute sa variété et donne une piste qui, sans dire directement qui il est ni où il se trouve, comme dans une partie de ping-pong, fait rebondir l'enquête et circuler géographiquement. Elles vont ainsi d'une part entretenir le suspense, et d'autre part enrichir les points de vue, de l'intérieur comme de l'extérieur.

51 Au niveau diégétique, le suspense est maintenu quant au fait pour Lex de trouver ou pas Sniper, de résister aux filatures et attaques des deux détectives-hommes de main qu'elle surnomme Bigote Rubio (" Moustache Blonde ») et Cara Flaca ( Face de Rat »), lancés sur sa piste à l'instigation de Lorenzo Biscarrués.

52 Les personnages rencontrés peuvent se ranger dans deux catégories en suivant le schéma actanciel de Greimas ${ }^{43}$, les adjuvants et les opposants. Ne changeront de catégorie que les personnages de la seconde enquête, qui d'opposants deviendront adjuvants par un retournement de situation vers la fin du roman, lorsqu'il s'avère que Lex poursuit le même objectif que Biscarrués, se venger de Sniper à titre personnel.

53 Mais jusque-là les choses sont simples au niveau narratif, marquant par là quel est l'objectif principal : voir et comprendre. Toutes les personnes rencontrées par Lex le sont sur recommandation de quelqu'un proche du monde du graffiti ou de l'art urbain et concourent à permettre d'en prendre la mesure. C'est même le cas de l'inspecteur chef Pachón, du Groupe sur les graffeurs de la Police Judiciaire de Madrid. S'il pourrait a priori être considéré comme un opposant à ce monde, il s'avère en réalité en prise directe avec le milieu dont il apprécie le mode d'expression tout en cherchant à en limiter les excès, brisant ainsi l'équation illégalité/ vandalisme/ absence de qualité.

54 Les personnages rencontrés dressent un portrait de la variété de cet univers. Ils peuvent se regrouper en deux catégories, si nous nous en tenons aux deux mondes définis par l'épigraphe : 


\section{l'inspecteur de police, à Madrid: Luis Pachón}

le directeur du département de conservation du patrimoine, à Lisbonne : Caetano Dinis

la spécialiste d'histoire de l'art, membre de la Fondation Salgari liée aux questions d'art urbain, à Vérone: Giovanna Sant'Ambrogio

le professeur d'université et critique d'art moderne dans la presse, à Rome: Paolo Taccia

à la frontière entre ces deux univers, un chauffeur de taxi de Naples, le conde Onorato ; son métier lui octroie la liberté de circulation dans la ville, et lui donne accès à tous ses habitants.

respect dans un monde en général masculin, avec un refus du "graffiti de genre ». S'introduit ici une ouverture vers la problématique générique, car il leur aura fallu trouver une lettre asexuée et non ronde, comme souvent l'est l'écriture des filles. Leur positionnement : « Nous avons accepté la légalité pour nous payer l'illégalité » (LPF, p. 97 ${ }^{47}$ ), ce qui les fait aller de la personnalisation d'objets à vendre, jusqu'à des expositions de leurs œuvres. 
course»(LPF, p. 169), rivalité entre bandes pour des questions de territoire ou de prestige.

$\mathrm{Au}$ terme de tous ces croisements, le dénouement se profile en deux temps lorsqu'enfin auront lieu les rencontres avec Sniper :

J'ai vu aussi un de ces sourires pour lesquels il faut avoir deux fois vingt ans avant que la vie ne vous le dessine ainsi sur les lèvres et dans le regard. Et alors, comme une révélation brutale, j'ai su que j'avais rencontré Sniper (LPF, p. 20948).

Celles-ci ont été préparées par des contacts avec l'écrivain de rues. Dans un premier temps, Lex lui a écrit une lettre dans laquelle figure ce qui semble être un manifeste pour l'art urbain par une série d'affirmations à la limite du sophisme telles que : "l'art actuel ne peut être que libre, l'art libre ne peut être réalisé que dans la rue, l'art dans la rue ne peut être qu'illégal, et l'art illégal se meut sur un terrain étranger aux valeurs qu'impose la société moderne", pour culminer par une sentence en forme de vérité absolue «l'œuvre d'art est au-dessus des lois de la société et de la morale de son temps » (LPF, p. $\left.116^{49}\right)$. Après des péripéties diverses, elle le rencontre et lui propose une sortie ensemble: « Toi et moi. Un mur adéquat dans un lieu adéquat. Dangereux, comme ceux où tu envoies les gens se suicider... Se faire... Comment disais-tu déjà? Ah, oui. Se faire jeter du vitriol en plein visage. » (LPF, p. 24950), ce qui est le titre du dernier chapitre, chacun marquant de manière cryptique ce qui apparaît plus loin comme une prise de position.

Comme on l'aura compris, tous ces contacts sont l'occasion de faire un bilan des différents positionnements autour de la question de l'art.

Pour Sniper, évoquée à plusieurs reprises, la critique de l'art contemporain est constante. Argumentée, pétrie de références culturelles, soutenue par des exemples érudits page après page, elle devient un manifeste non plus dans des discours rapportés mais par sa voix directement: «Les grecs ont exprimé l'harmonie [...]. Aujourd'hui, pourtant, l'art nous rend plus... [...] Stupides?» (LPF, pp. 244-5 $5^{51}$ ). Les instruments sont des preuves: "Depuis toujours, les artistes ont utilisé des instruments qui jouaient le rôle de moteurs (...). Moi, je me sers de vitriol. Au figuré, bien sûr. Ou pas tellement. » (LPF, p. 268 ${ }^{52}$ ). La focalisation interne lui permet de développer son point de vue : «Je ne fais pas de l'art conceptuel, ni de l'art conventionnel. Je fais de la guérilla urbaine» (LPF, p. 216 ${ }^{53}$ ). Les exemples foisonnent pour dire longuement le rejet de l'art et de son monde et affirmer une idéologie: «Les rues sont l'art... L'art n'existe que pour éveiller nos sens et notre intelligence, et pour nous lancer un défi. [...] L'art n'est pas un produit, mais une activité » $\left(L P F\right.$, p. $\left.218^{54}\right)$. Paradoxalement, le rejet de l'art aboutit à une revendication du statut d'art, et de plus est élevé au rang d'unique art vivant : « Le graffiti est la guérilla de l'art » (LPF, p. 219). Une valorisation de l'œuvre elle-même en affirme l'essence :

le graffiti est l'œuvre d'art la plus honnête, parce que celui qui le fait n'en profite pas. [...] Et même si plus tard l'artiste finit par se vendre, l'œuvre faite dans la rue y reste et ne se vend jamais (LPF, p. 22155).

Au-delà d'une évidente radicalisation du propos, le personnage propose une réflexion intéressante sur l'affirmation de la différence entre l'artiste et l'œuvre. Et enfin pendant l'ultime intervention, a lieu la conversation finale sur « un art qui en soit vraiment un "(LPF, p. 264 et suite). Il s'agit de transformer la stupidité d'une époque en œuvre d'art ; le danger est le seul à même d'octroyer sérieux et authentification de l'action dans une tentative de faire apparaitre l'absurdité, de donner de la gloire, de la dignité et de la responsabilité ; enfin, de rendre le sentiment du réel... 

narration découvre que Lita, amante de Lex, est l'une des victimes innocentes de la manipulation de Sniper, on comprend le coup de théâtre final. La narratrice, partie en opération avec lui, lui plante son couteau dans les reins par deux fois en affirmant: "C'était moi, l'assassin». L'enquête double se révèle avoir été triple, la narratrice poursuivant la recherche de Sniper pour son propre compte. Et tous, le coupable devenu victime, les commanditaires des deux autres enquêtes, et le lecteur, comprennent qu'ils s'étaient trompés. Le graffeur sera retrouvé écrasé par le métro et on ne pourra que conclure à un accident. Sa compagne, vue puis rencontrée, sera finalement la seule qui comprendra ce qui s'est passé et éveillera l'ombre vague d'un remord en Lex, mais pas de nature à réorienter l'axiologie du texte. En effet Lita, graffeuse, est érigée comme contrepoint de Sniper, sorte de prise de position finale :

Elle croyait à tout ce qu'on peut croire à dix-huit ans. [...] Et, figure-toi, elle écrivait sur les murs. Elle sortait la nuit dans les rues pour y laisser le témoignage du regard plein de tendresse qu'elle projetait sur le monde. Pour y affirmer son humble nom, livrant son propre combat, à sa manière... [...] Ce terrible désespoir qui l'assaillait trop souvent, toute cette émouvante innocence trahie par l'injustice aveugle de la vie réelle. (LPF, pp. 268-27061).

Il est clair devant un tel positionnement que le graffiti, devenu art de rue et performance, franchit avec Sniper aux commandes une nouvelle étape, l'organisation de montages (ou murs baise chaque endroit qu'il foule ou qu'il tiennent pour Dieu, le Fils et le Père réunis. » (LPF, p. $26^{56}$ ).

Un bombardement continu d'images destinées à manipuler celui qui les regarde a effacé les frontières entre le réel et l'artificiel... Mon art restitue le sentiment du réel avec tout ce qu'il comporte de tragédie. (LPF, p. 266 ${ }^{57}$ ).

Ce mot qui porte un nom de putain ? [...] L'art moderne n'est pas de la culture, il est

Avec un jusqu'au-boutisme désespéré, il hurle sa volonté d'apocalypse : "Je ne cherche pas un monde meilleur. [...] Je cherche à détruire notre temps. » (LPF, p. 26759). qui avait créé le socle dela no perçoit une inflexion dans le jugement de valeur implicite avec le refus de l'extrémisme idéologique du personnage. Auparavant, le graffeur El Topo avait déjà très tôt proposé un être un plan pour faire augmenter sa cote dans un monde de la rue rapide où l'on disparaît aussi vite qu'on apparaît. Et la narratrice avait également mis en évidence l'orgueil de « te vanter à tes propres yeux de ton manque de prétention. » (LPF, p. $\left.243^{60}\right)$.

champ lexical n'est plus celui de la guerre mais celui de la mélancolie, la tendresse, et, même si celui-ci est hostile, le besoin d'être au monde. 


\section{Une géographie internationale du graffiti : Madrid, Lisbonne, Vérone, Naples}

76 Par la circulation entre les personnages, le texte avait voulu évoquer une culture globale du graffiti. La diégèse, qui se construit autour du niveau maximal des productions de Sniper, celles où il n'intervient qu'à distance, fait apparaître deux éléments supplémentaires révélant une conception globale d'une production locale: un fonctionnement désincarné, délégué par le biais des nouveaux media et revendiqué par le même canal, ainsi qu'une internationalisation des sources et des circuits.

Le personnage de Sniper, dans sa dernière phase, n'est intéressé que par l'exploit physique et sportif qu'il parvient à déclencher par des événements qu'il convoque, toujours forts et spectaculaires et dépassant les limites du simple graffiti ; idea lanzada, actuación, acontecimientos en toda regla, sont les termes employés sans qu'il soit possible de les caractériser davantage. Il mobilise la communauté internationale des écrivains de rue et stimule ses followers par la valeur performative de l'acte. La réalisation n'est en fait que la fin du processus. Si la mise en œuvre est large, la conception, qui comprend des études et maquettes préalables aux actions ou des relevés d'intentions, reste artisanale, dans un fond de garage: " cela n'avait pourtant rien d'un atelier d'artiste. C'était un laboratoire génial de guérilla urbaine » (LPF, p. $\left.241^{62}\right)$. Cela dénote un véritable travail, de l'ébauche à la genèse de l'acte, chacune de ces étapes représentant des millions d'euros potentiels dans le cas d'une commercialisation. Comme le suggère Norman Mailer, «l'ego est un capital transformable en monnaie par l'emploi du nom ${ }^{63}{ }^{\text {». }}$

Certes le danger est augmenté par la criminalisation du graffiti qui concourt à prendre des risques, surtout au regard de la vitesse d'exécution. C'est exactement ce qui est recherché par Sniper, l'imagination en la matière ne pouvant connaître de limites et dépassant la question de l'interdit. Différents exemples installent la gradation, mêlant réalisation réelles et fictionnelles, dans une approche du possible.

D’une célébrité obtenue vers 1995, avec l'invention du palancazo («le coup du signal d'alarme ») qui consiste à monter dans un train à six ou sept, puis tirer le signal d'alarme, bondir à l'extérieur quand le train s'arrête et dessiner au nez et à la barbe des voyageurs, pour une plus grande visibilité, il recherche ensuite les cibles internationales : pont de la Tamise à Londres, pont Metlac de Veracruz au Mexique... En conséquence, il emporte l'adhésion de dizaines de jeunes décidés à risquer leur vie, avec surtout leur action enregistrée sur téléphone portable et postée sur les réseaux. Car la stimulation, l'adrénaline produite par la transgression, la possibilité de se mesurer à soi-même, de montrer son courage entrent dans une spirale où la reconnaissance du milieu est essentielle.

80 Les derniers exploits dans ces intervenciones sont décrits longuement pages 261 et suivantes. De la même manière sont développés les détails sur l'intervention qui a causé la mort de Lita. Le lecteur est emporté par la pluralité des situations évoquées dans une surenchère où le jeu stimule et l'originalité suscite l'adhésion.

81 L'auteur semble lui-même emporté dans l'ivresse de l'invention, comme lorsqu'il fait évoquer à Sniper son projet suivant: lancer une armée de graffeurs pour peindre le bateau de la compagnie Costa échoué en Italie, «monument à l'irresponsabilité, l'inconscience et la stupidité humaines » (LPF, p. 22064). 

sorte, le lecteur peut suivre son raisonnement lorsqu'il définit sa conception extrémiste de l'art. Mais apparaît alors dans le dernier chapitre ce mépris pour les autres humains, et pas seulement pour la société qui dysfonctionnerait :

$\mathrm{Tu}$ crois peut-être que le terroriste aime l'humanité pour laquelle il dit lutter ? (...) Nous ne méritons pas de survivre (...) nous méritons une balle dans la tête, un par un. / Le franc-tireur patient. (LPF, p. 26465).

Explicitant enfin le titre en expliquant son nom, le protagoniste confirme cyniquement que les décès font partie de l'intervention : «Ils la transforment en une affaire sérieuse. Ils l'authentifient. » (LPF, p. 264), tout en maintenant une différence moralement discutable entre incitation et assassinat: «Je prépare seulement l'absurde. Ce sont les autres qui, à leurs risques et périls, remplissent les pointillés. " (LPF, p. 26566). L'absence de causalité juridique dérive du fait que lui-même n'agit pas et n'accompagne pas; il choisit et commente les objectifs sur les réseaux sociaux puis chacun fait son choix. Cela s'avère possible par un besoin de reconnaissance commun à tous et parachevé par la couverture médiatique.

Internet occupe ainsi une place primordiale; il est l'aiguillon, le mécanisme de coordination, le vecteur d'annonce des réalisations et la conservation de la trace. Avec la vidéo ou la photographie, à l'origine destinée à déposer une preuve du haut-fait dans l'album-photo de chacun, la pièce va a être diffusée et publicisée, et donc pérennisée :

Il y a des règles, des codes. Tout le monde sait que ce mur est ce qu'il est. Il faut des témoins pour prouver que le travail a été fait comme il doit l'être. Mettre la vidéo sur Internet et tout le reste. Chez les auteurs de graffitis, le seul mot qui compte est réputation. C'est pour elle qu'on fait tout : la réputation. (LPF, p. $\left.261^{67}\right)$.

T.S. Eliot a démontré le «nécessaire rattachement de l'artiste à sa communauté, et le désir d'atteindre le plus grand nombre de ses membres $^{68} »$. Ici, il ne s'oppose pas mais s'ajoute à ce « mythe romantique du génie individuel, pour qui le retrait hors de la société et le mépris du succès sont constitutifs de la vision artistique ${ }^{69}$ » selon la formulation de Richard Shusterman. Dans le cas de l'art urbain, il y a dualité, voire multiplicité, du public aux réactions opposées. Internet et les réseaux sociaux donnent ainsi accès à un public digital d'initiés, mais pas uniquement, tout comme dans la rue. Il offre une célébrité instantanée, mais avec potentialité de durée.

Progressivement on parvient à la constitution de quelque chose de commun, à travers une juxtaposition d'actions singulières qui dépassent les frontières. Une géographie du graffiti se fait jour, internationalisant le propos, de Madrid à Lisbonne, Vérone ou Naples. Les productions restent spécifiques mais s'étendent sous une forme rhizomatique. Les échanges rappelés par le texte incluent le mélange des styles -le manga japonais, l'influence de la culture new-yorkaise et du hip hop-, se faisant toujours plus larges, créant des mouvances connues comme graffiti américain ou européen. Des manifestations internationales y sont consacrées comme les Meeting of Styles de Wiesbaden ou à la Tate Modern de Londres donnant un prestige tout à fait international. Une extension planétaire est remarquable, parallèle à la mobilité contemporaine, dans laquelle toute expression est assimilable par d'autres, sans être identique. Les villes se définissent ainsi selon les manifestations scripturales et picturales qu'elles recèlent, prenant une identité par leurs manifestations visuelles.

87 Dans le cadre de La patience du franc-tireur, la géographie textuelle se dessine de chapitre en chapitre, sans retour en arrière. À chaque étape, il faudra des guides locaux, 
appelés des " explorateurs indigènes », pour s'approprier la géographie d'une ville et en connaitre les potentiels enjeux.

L'itinéraire commence à MADRID, du fait de la nationalité des deux personnages principaux. L'invention du palancazo et du jeu sur l'épaisseur des traits sont relevés comme étant « la contribution de Madrid à la culture planétaire du graffiti » (LPF, p. 69).

Il entraîne ensuite les personnages et les lecteurs vers une LISBONNE, évoquée comme la capitale mondiale du graffiti, où « les graffeurs sont très bien organisés » (LPF, p. 53), ville « au prestige international comme capitale du graffiti » (LPF, p. 83). Entremêlant éléments référentiels avérés, considérations générales et exemples fictionnalisés, le texte fourmille de descriptions, exemples de performances, enjeu des négociations artistes-municipalité, fonctionnement, intégration aux circuits touristiques et commerce. Il réalise un circuit urbain, avec exemples d'œuvre et topographie sur plus de six pages de suite (p. 84-90). S'il y a volonté de rompre le lien graffiti-vandalisme, le texte relève les potentiels excès, comme lorsque la fiction intègre l'expression d'une centaine de graffeurs qui ont barbouillé la ville d'un œil noir, hommage à Saramago, à l'instigation de Sniper, avec plus de 2000 pièces. Face à cela, une loi du silence, sorte d'omerta qui exclut les non-initiés. Allant plus loin dans cette voie, est évoquée l'idée d'une peinture ultra-violette visible seulement avec des instruments d'optique, qui dessinerait ainsi « une Lisbonne secrète, invisible pour le profane » (LPF, p. 91).

Dans une sorte de jeu narratif, un message de Sniper annonce fin du chapitre 3, « Movida en Italia ", réorientant le voyage de Lex. On quitte alors la péninsule ibérique pour rejoindre l'Italie, dont deux lieux majeurs seront explorés.

91 VERONE tout d'abord avec « une des plus originales interventions d'art urbain illégal réalisées en Europe » comme annoncé par les media italiens ( $L P F$, p. $\left.107^{70}\right)$. Sniper s'est introduit dans la cour de la maison-musée de la légende de Shakespeare, haut-lieu du tourisme véronais pour attirer l'attention, mais aussi convoquer là des centaines de graffeurs pour un autre événement mystérieux à venir.

NAPLES enfin, qui est dans le roman la base arrière de Sniper. Seule ville orientale, à l'exception d'Istanbul, dit-il, qui se trouve géographiquement en Europe, elle se déploie largement à partir du chapitre 6 et jusqu'à la fin ; elle est symbole de la décomposition d'un monde qu'il trouve stupide car sûr de lui-même jusqu'au suicide.

On y découvre la complexité de sa vie sociale et politique dans laquelle l'expression urbaine s'intègre. C'est aussi l'espace des temples du graffiti. L'un, écho du renouveau de l'art, quand des graffeurs sont invités à décorer une chapelle en rénovation. L'autre, secret encore, lorsque Lex descend sous terre avec Sniper, et découvre un tunnel qui mène au métro de Naples :

une galerie spectaculaire décorée jusqu'à saturation, peinture sur peinture, depuis de simples tags au marqueur jusqu'à des fresques compliquées faites à l'aérosol, se superposant dans un dépoilement impressionnant de traits et de couleurs. - C'est notre Chapelle Sixtine... Plusieurs générations de graffeurs sont passées ici. (LPF, p. $\left.257^{71}\right)$

94 C'est donc également une ville pour la mémoire, quand, quelques siècles après une catastrophe ou une guerre nucléaire, « les archéologues, impressionnés, découvriront cet endroit » (LPF, p. 257), assurant une postérité à l'éphémère.

Une carte européenne apparaît donc sous nos yeux, et des allusions moins poussées sont faites aux États-Unis et au Japon. La présence de ces références géographiques 
extratextuelles ne se limite toutefois pas à une volonté de certification. Car le traçage des actions pouvant avoir existé dans le hors-texte n'aurait qu'un intérêt relatif. Bien plus utile nous semble l'imbrication avec une fiction qui n'a d'imaginaire peut-être que le fait de ne pas avoir été encore réalisée, marquant ainsi le refus de cloisonner et de limiter à l'existant une expression urbaine par définition sans limite.

C'est ainsi que des espaces urbains évoqués sont aussi symboliques. Ainsi, face aux villes exécrées, car « empoisonnées » par salissures, fumée, pollution, affiches, cartes de crédit, publicités et caméras, la ville rêvée de Sniper voudrait être :

Une ville de couleurs, d'incitations, de phrases, de pensées qui feraient réfléchir, une ville d'authentiques messages de vie. Une espèce de fête urbaine où tout le monde serait invité et dont personne ne serait jamais exclu... (LPF, p. 21972).

C'est à ce titre que ville et femme aimée ne font qu'un ; la compagne napolitaine de Sniper, son arrière-garde, la seule qui le retient dans son cynisme désespéré : « Elle fait partie de la ville [...]. Comprise avec le quartier » (LPF, p. 243 $\left.{ }^{73}\right)$.

\section{Conclusion}

Dans El francotirador paciente, Arturo Pérez-Reverte se plonge dans la ville européenne. Derrière ses personnages, il endosse un rôle militant en interaction avec l'espace urbain défiguré par la publicité et dévasté par la pollution ${ }^{74}$. L'auteur affirme la nécessaire participation des jeunes à la construction d'un paysage urbain, pour un double bénéfice, donner à celui-ci l'oxygène qui lui fait défaut et exister dans un monde qui ne leur laisse pas beaucoup de place. En mettant en évidence une valeur symbolique de l'espace, le roman livre une épique du graffiti, avec une éthique mais des limites sans cesse repoussées. Au-delà de la mythification initiale et naïve du graffeur, le texte propose surtout d'aider à regarder. Comme le dit Thierry Paquot, « la ville est potentiellement écriture [...] mais pour être vraiment visible et lue, cette ville devenue écriture réclame la voix, c'est-à-dire les pas du marcheur, de l'explorateur, du découvreur-lecteur ${ }^{75} \ggg$. Et c'est dans ce sens que ce regard littéraire espère apporter sa contribution aux réflexions en cours sur l'art urbain.

\section{BIBLIOGRAPHY}

Figueroa, Fernando, et Gálvez, Felipe, Firmas, muros y botes, Madrid, auto-édition (http:// www.firmasmurosybotes.com), 2014.

Greimas, Algirdas, Sémantique structurale, Paris, Larousse, 1966.

Jouve, Vincent, L'effet-personnage dans le roman, Paris, Presses universitaires de France, Collection «Écriture », 1992

Mailer, Norman, « La foi du graffiti » in Morceaux de bravoure, (Boston, Little, Brown and Company, 1982), trad. française : Paris, ed. Robert Laffont, coll. Pavillons poche, 2012, pp. 217-254. 
Manzano, Fátima G., « El mensaje oculto tras los grafitis », elmundo.es, http://www.elmundo.es/ f5/2015/10/21/55a8ddf1e2704e982c8b457a.html (actualisé 20/10/2015)

Paquot, Thierry, Des corps urbains - Sensibilités entre béton et bitume, Paris, éd. Autrement, 2006.

Shusterman, Richard, L'art à l'état vif-La pensée pragmatiste et l'esthétique populaire, Paris, éd de Minuit, 1991 (trad.) «4. Forme et funk : le défi esthétique de l'art populaire » (pp. 137-82)

\section{NOTES}

1. Première édition chez Alfaguara en 2013. L'édition espagnole utilisée ici est: Arturo PérezReverte, El francotirador paciente, Barcelona, Pengin Random House Grupo Editorial, coll. Debolsillo, 2015. Les citations espagnoles qui en sont tirées figurent en notes, suivies de EFP et de la page.

2. S'agissant de l'analyse d'un texte littéraire au sein d'une réflexion pluridisciplinaire, il nous a paru essentiel de pouvoir suivre la mise en mots de ces enjeux. Pour cela un nombre important de citations est convoqué. Pour plus de légèreté, elles sont directement suivies dans le corps du texte de la page entre parenthèses, ainsi que de $L P F$, en référence la traduction française de François Maspero, La patience du franc-tireur, Paris, éditions du Seuil, 2014. L'appel de notes renvoie au texte original.

3. Vincent Jouve, L'effet-personnage dans le roman, Paris, Presses universitaires de France, Collection « Écriture », 1992.

4. « alguien encargado de localizar autores y libros interesantes. Una especie de rastreador culto, cualificado, con buen olfato », EFP, p. 20.

5. « a medio camino entre Banksy y Salman Rushdie... », EFP, p. 23.

6. « marca registrada, y punto : Sniper. El francotirador solitario. », EFP, p. 23.

7. " yo lo consagro para siempre y hago entrar su obra en el círculo de los dioses », EFP, p. 24.

8. Norman Mailer, "La foi du graffiti ", in Morceaux de bravoure, (Boston, Little, Brown and Company,1982), trad. française : Paris, ed. Robert Laffont, coll. Pavillons poche, 2012, p. 217.

9. «Erase una vez una raza especial de personas/ llamadas escritores de grafiti./ Pelearon una batalla contra la sociedad./El resultado todavía se desconoce. ", EFP, p. 9.

10. "lobos nocturnos, cazadores clandestinos de muros y superficies, bombarderos sin piedad que se movían en el espacio urbano (...) merodeando con sus aerosoles », EFP, p. 9.

11. " por las mochilas y el aspecto » / « el gesto de pintar algo », EFP, p. 9.

12. « sólo se ascendía por méritos ganados en solitario o en pequeños grupos, imponiendo cada cual su nombre de batalla con esfuerzo y constancia, multiplicándolo hasta el infinito por todos los rincones de la ciudad ", EFP, pp. 9-10.

13. " plebeyos, simple infantería. El escalón más bajo de su tribu urbana. Parias de una sociedad individualista », EFP, p. 9 / « obras de consagrados, de reyes callejeros », EFP, p. 10.

14. « en un muro, en un vehículo, en el cierre metálico de una tienda (...) buscando juntos huecos o piezas ajenas en paredes saturadas, fábricas abandonadas del extrarradio e instalaciones ", EFP, p. 9.

15. « con apresuramiento y poco arte, más cantidad que calidad », EFP, p. 25.

16. « un mundo hecho de códigos, reglas no escritas y símbolos para iniciados », EFP, p. 10.

17. « siguen unas reglas, técnicas y códigos de colores que sólo la comunidad conoce al detalle. El morado y el violeta para los movimientos feministas, el negro para los anarquistas, el azul para aquellos que se sienten identificados con la derecha », in Fátima G. Manzano, "El mensaje oculto tras los grafitis", elmundo.es.

http://www.elmundo.es/f5/2015/10/21/55a8ddf1e2704e982c8b457a.html (actualisé 20/10/2015)

18. " pisarle el tag a alguno de los grandes », EFP, p. 10. 
19. « un tachado sobre una firma ajena era siempre una declaración de guerra ; una violación de nombre, territorio, fama de otros ", EFP, p. 10.

20. « Los duelos eran frecuentes, EFP, p. 10.

21. «AKTJ : «Adivina Kién te jode», EFP, p. 12. Cf l'argot (joder) et la déformation orthographique (kién pour quién).

22. Norman Mailer « La foi du graffiti », op. cit., p. 225 et 231.

23. « poco atentos al estilo, sin respetar nada ni a nadie », EFP, p. 10.

24. Norman Mailer, op. cit.., " pas seulement le désir infantile de voir passer son nom en lettres assez grandes pour hurler l'ego d'un bout à l'autre de la ville, non (...) revenir à une sensation plus primordiale de l'existence » p. 254.

25. « el nombre de Nadie multiplicado hasta el infinito, EFP, p. 30.

26. " olor a gloria urbana, a libertad ilegal, a fama dentro del anonimato. A chorros, bum, bum, bum, de adrenalina ", EFP, p. 12.

27. « mucho más que una simple obra de grafitero común. Era una pieza en toda regla », EFP, p. 13.

28. Richard Shusterman, L'art à l'état vif - La pensée pragmatiste et l'esthétique populaire , Paris, éd de Minuit, 1991 (trad.), « 4. Forme et funk : le défi esthétique de l'art populaire» (pp. 137-82), p. 176.

29. Plusieurs titres de chapitres, en particulier les $1,2,5,7,9$, ont valeur de manifestes: 1 . Las ratas no bailan claqué ; 2. Si es legal no es grafiti ; 3. Los grafiteros ciegos ; 4. El balcón de Julieta ; 5. Esto es lo que no soy ; 6 . El sicario culto ; 7. Treinta segundos sobre Tokyo ; 8 . El cazador y la presa ; 9. Acido en la cara.

30. «así que era eso (...). La excitación intelectual, la tensión física, el desafío a tu propia seguridad, el miedo dominado por la voluntad, el control de sensaciones y emociones, la inmensa euforia de moverse en la noche, en el peligro, transgrediendo cuanto de ordenado el mundo establecía, o pretendía establecer. Moviéndose con sigilo de soldado en los estrechos márgenes del desastre. En el filo incierto de la navaja », EFP, p. 224.

31. « agresivo carácter individualista, callejero, transgresor y clandestino », EFP, p. 48.

32. " a medio camino entre la letra burbuja y el estilo salvaje, letras rojas como salpicaduras y una característica mira teléscopica de rifle sobre el punto de la i »,EFP, p. 39.

33. " puestas entre las letras como apartándolas para invadir, amenazadoras, el espacio urbano ", EFP, p. 39.

34. " sus personajes eran siempre alusiones originales, atrevidas, con mucho sentido del humor, a clásicos famosos : una calavera de Gioconda con estética punki (...) », EFP, p. 39.

35. « el famoso grafitero de Bristol, empezaba a hacer algo parecido en Inglaterra. », EFP, p. 41.

36. « arte urbano nuevas tendencias y demás », EFP, p. 42.

37. « Lo de artista sonaba a desdén gremial », EFP, p. 187.

38. «Hasta el arte callejero lo han convertido los ayuntamientos en parque temático (...). Ese imbécil concepto de participación pública, tan socialmente correcto, acabó siendo una simple diversión más (...). Pero yo demuestro que, de diversión nada. Y que a veces la vida va en ello. ", EFP, pp. 267-8

39. «El Evangelio en once putas palabras. En un costado del coche escribió : Si es legal no es grafiti. Y en otro : Las ratas no bailan claqué. », EFP, p. 43.

40. «El poder siempre intenta domesticar lo que no puede controlar », EFP, p. 81.

41. «Diseño fundas de cedés, tatuajes, customizo ropa, decoro con los gobetti alguna tienda de por aquí... Me las arreglo. », EFP, p. 266.

42. " Eso es lo que no soy ", EFP, p. 153.

43. Algirdas Greimas, Sémantique structurale, Paris, Larousse, 1966.

44. Peut-être une référence à Daniel « El Topo » Rivero, né au Venezuela et connu pour ses écrits à Gand en Belgique. 
45. « olor civilizado, resignación para comer caliente », EFP, p.72.

46. http://www.eldiario.es/cultura/Muelle-sfhir-graffiti-grafiti-reverte-arguello-remebe-artistaurbano-muerte-aniversario_0_403860234.html (consulté le 5/11/2015)

47. « Aceptábamos lo legal para pagar lo ilegal », EFP, p. 105.

48. « vi una sonrisa de las que hace falta tener dos veces veinte años para que la vida te la defina en la boca y en la mirada. (...) como en una revelación brutal, supe que había encontrado a Sniper », EFP, p. 228.

49. « el arte actual sólo puede ser libre, el arte libre sólo puede ser ilegal, y el arte ilegal se mueve en un territorio ajeno a los valores que la sociedad impone » / «la auténtica obra de arte está por encima de las leyes sociales y morales de su tiempo », EFP, p. 127.

50. « Tú y yo. Una pared adecuada y un lugar adecuado. Peligroso, como esos adonde envías a la gente a suicidarse. A hacer... ¿Cómo dijiste antes ? Ah, sí. A tirar ácido a la cara. », EFP, p. 271.

51. «Los griegos marcaron la armonía (...) Ahora el arte nos hace más ... (...) ¿Estúpidos ? », EFP, p. 266.

52. « Desde siempre, los artistas han utilizado instrumentos, motores (...). Yo uso ácido. Figurado, claro. O no tanto. », EFP, p. 268.

53. «Yo no hago arte conceptual, ni arte convencional. Yo hago guerrilla urbana », EFP, p. 237.

54. « Las calles son el arte... El arte sólo existe para despertarnos los sentidos y la inteligencia y para lanzarnos un desafío. (...) el arte no es un producto, sino una actividad », EFP, p. 239.

55. « es la obra de arte más honrada, porque quien la hace no la disfruta. (...) Y aunque más tarde el artista se acabe vendiendo, la obra hecha en la calle sigue allí y no se vende nunca ", EFP, p. 242.

56. " vuelve locos a grafiteros de varias generaciones (...) en plan secta. Que lo tienen por Dios y su bendito padre. ", EFP, p. 28.

57. «Un bombardeo continuo de imágenes destinadas a manipular al espectador ha borrado las fronteras entre lo real y lo falso... Lo mío devuelve con su tragedia el sentimiento de lo real », EFP, p. 290.

58. « esa palabra con nombre de puta (...) el arte moderno no es cultura, sólo moda social (...) una ficción para privilegiados millonarios y para estúpidos (...) Es un comercio y una falsedad absoluta. », EFP, p. 291.

59. « No busco un mundo mejor. (...) Busco destruir nuestro tiempo. », EFP, p. 292.

60. « Presumir ante ti mismo de tu poca presunción », EFP, p. 265.

61. «Creía en todo lo que puede creerse a los dieciocho años. (...). Y era escritora de paredes, fíjate. Salía de noche a la calle para dejar constancia allí de la mirada que, desde su ternura, proyectaba sobre el mundo. Para afirmar su humilde nombre en él, librando su propia lucha, a su manera ... (...) Esa singular desesperación que la acometía a veces, toda su conmovedora inocencia traicionada por la imprecisa injusticia de la vida real », EFP, p. 293.

62. « no era, desde luego, un taller de artista. Era un genial laboratorio de guerrilla urbana », EFP, p. 263.

63. Norman Mailer « La foi du graffiti », op. cit., p. 218.

64. « monumento a la irresponsabilidad, la inconsciencia y la estupidez humanas », EFP, p. 241.

65. «Acaso crees que el terrorista ama a la Humanidad por la que dice luchar? (...) No merecemos sobrevivir (...) merecemos una bala en la cabeza, uno por uno. / El francotirador paciente. », EFP, p. 289.

66. "Yo sólo planteo el absurdo. Son otros los que, a su costa, rellenan la línea de puntos », EFP, p. 289.

67. «Hay reglas, códigos. Todo el mundo sabe que esta pared es lo que es. Se necesitan testigos para probar que se hizo como es debido. Colgar el vídeo en Internet y cosas así. Entre los escritores de graffiti, la única palabra que cuenta es reputación. Por eso se hace todo: por reputación. », EFP, p. 285. 
68. T.S. Eliot, The use of poetry and the use of criticism, Londres, Faber, 1954, pp. 152-53 ; cité par R. Shusterman, op.cit., p. 170.

69. R. Shusterman, op.cit., p. 170.

70. « una de las más originales intervenciones de arte urbano ilegal realizadas en Europa », EFP, p. 117.

71. « una espectacular galería decorada hasta la saturación, pintura sobre pintura, desde simples tags con rotulador hasta piezas complicadas hechas con aerosol, superpuestos unos a otros en un despliegue espectacular de trazos y color. - Es nuestra capilla Sixtina... Varias generaciones de escritores pasaron por aquí. », EFP, p. 281.

72. «Una ciudad de colores, de impactos, de frases, de pensamientos que harían pensar, de mensajes reales de vida. Una especie de fiesta urbana donde todos estuvieran invitados y nadie excluido jamás... », EFP, p. 240.

73. « va incluida en la ciudad (...) Viene con el barrio », EFP, p. 265.

74. Le graffeur Muelle disait: «Entendemos nuestro trabajo como una herramienta para transformar la calle y crear vínculos entre las personas », cité par Fátima G. Manzano dans «El mensaje oculto tras los graffitis ", elmundo.es,

http://www.elmundo.es/f5/2015/10/21/55a8ddf1e2704e982c8b457a.html (actualisé 20/10/2015)

75. Thierry Paquot, Des corps urbains - Sensibilités entre béton et bitume, Paris, éditions Autrement, 2006, p. 72.

\section{ABSTRACTS}

Avec ses outils propres, le roman de l'espagnol Arturo Pérez-Reverte, El francotirador paciente (2013) - La patience du franc-tireur - apporte une contribution à la tentative de déterminer des constantes définitoires, évolutions temporelles et spécificités spatiales de l'art urbain. Le processus narratif construit l'analyse des enjeux et modalités de ses expressions - fonction, action, production -, dans une circulation labyrinthique dans des villes européennes mettant en évidence la valeur symbolique des espaces. À partir d'une triple trame d'investigation, l'œuvre littéraire livre une épique du graffiti aux limites sans cesse repoussées et suggère une éthique. Au-delà de la mythification initiale du graffeur, le texte propose surtout de regarder et de questionner.

Con sus propias herramientas, la novela del español Arturo Pérez-Reverte, El francotirador paciente (2013) lleva una aportación al intento de determinar constantes definitorias, evoluciones temporales y especificidades espaciales del arte urbano. El proceso narrativo construye el análisis de lo que está en juego y de las modalidades de sus expresiones - función, acción, producción -, en una circulación laberíntica por ciudades europeas poniendo de relieve el valor simbólico de los espacios. A partir de una triple trama de investigación, la obra desvela una épica del grafiti cuyos límites siempre se ensanchan, y sugiere una ética. Más allá de la mitificación inicial del grafitero, el texto sobre todo incita a mirar y cuestionar. 
INDEX

Geographical index: Espagne

Chronological index: XXIème siècle

AUTHOR

CHRISTINE DI BENEDETTO

Université Nice Sophia Antipolis - UFR LASH - Espagnol - LIRCES 